\title{
Interprofessional Education Between Athletic Training and Advanced Practice Nursing Programs
}

\author{
Heather Hudson, EdD, LAT, ATC ${ }^{1}$ - Deborah Shirey, DNP, APRN, FNP-BC² - Meagan Soltwisch, DNP, APRN, FNP-C ${ }^{2}$. \\ Matthew Peterson, $\mathrm{PhD}^{3}$
}

Interest in interprofessional education (IPE) has increased over the past 30 years. As the health care industry changes its focus to more patient-centered care through collaborative practices, IPE in health care educational programs has become more predominate to meet this demand. The shift from solo practice to collaborative practice has evolved to reduce medical errors seemingly prevalent in health care because of poor communication and teamwork and to provide patients with holistic care. Research indicates greater positive outcomes when individuals and teams practice collaboratively compared with and individualistic approach.

According to the World Health Organization (WHO), interprofessional collaboration occurs when multiple health care workers from different disciplines work synergistically with the intent of deliver- ing the highest quality of care to patients, families, and communities. ${ }^{2}$ The ability to work collaboratively is difficult, and it takes time to build skills for working collaboratively to be successful. Organizations such as WHO, the Interprofessional Education Collaborative (IPEC), and discipline-specific organizations have called upon health care educational programs to include interprofessional collaboration as part of the curriculum through utilizing IPE. ${ }^{3,4}$ Therefore, agencies governing accreditation for many health care programs have started to mandate IPE as a part of curriculum standards.

IPE has varying definitions depending on the organization that defines it. WHO defines IPE as "when students from two or more professions learn about, from, and with each other to enable effective collaboration and improve health outcomes."2 Additionally, IPEC has developed

\section{AFFILIATIONS:}

'Department of Health, Human Performance, and Recreation, Robbins College of Health and Human Sciences, Baylor University, Waco, Texas

${ }^{2}$ Louise Herrington School of Nursing, Baylor University, Dallas, Texas

${ }^{3}$ Baylor University, Waco, Texas

\section{CITATION:}

Hudson H, Shirey D, Soltwisch M, Peterson M. Interprofessional education between athletic training and advanced practice nursing programs. Consultant. Published online May 24, 2021. doi:10.25270/ con.2021.05.00009

Received January 12, 2021. Accepted February 4, 2021.

\section{DISCLOSURES:}

This work was supported by a $\$ 5000$ research grant from The Academy for Teaching and Learning at Baylor University.

\section{CORRESPONDENCE:}

Heather Hudson, EdD, LAT, ATC, Baylor University, One Bear Place \#97313, Waco, TX 76798 (Heather_hudson@ baylor.edu)

4 core competencies to guide both the health care industry and educational programs in developing interprofessional practice and education. ${ }^{5}$ The 4 core competencies are (1) values/ethics for interprofessional practice, (2) roles/ responsibilities, (3) interprofessional communication, and (4) teams and teamwork. ${ }^{5}$ The premise behind employing IPE is to intentionally provide opportunities for students to authentically engage with each other to practice and build on the competencies necessary to work collaboratively. Research has demonstrated positive outcomes when students are exposed to IPE concepts, particularly in the areas of understanding their own roles and responsibilities as health care professionals functioning within a team. ${ }^{6-10}$ In addition, students gain awareness and understanding of the roles and responsibilities of other health care professionals, and there has been a positive increase in the communication that transpires among professionals because of IPE.

Historically, health care programs have instilled one or more competencies targeting a team approach to practice within curriculums. However, a true intentional focus on building interprofessional collaborative skills was not always apparent, valued, or possible.11-13 For example, athletic training programs have had to include some focus on a team approach to practice in their curriculums since 2012 when the athletic training professional standards for professional programs and the foundational behaviors of practice were published in the Athletic Training Edition Competencies. ${ }^{14}$ These 
standards require athletic training skills in order to graduate from the program. Even with these requirements, a study conducted in 2013 revealed that very few athletic training programs either participated in IPE activities or had the means to participate in IPE activities. ${ }^{15}$ It was not until the 2020 Standards for Professional Masters Programs that IPE and practice have become more focused. Nursing, on the other hand, has included interprofessional collaboration as a part of program curriculum requirements for more than 10 years. These competencies are reflected in the American Association of Colleges of Nursing (AACN) Essentials, which are published for baccalaureate, master's, and doctoral levels of nursing education. ${ }^{4,17-18}$

\section{OBJECTIVE}

IPE researchers have studied students' attitudes and readiness toward IPE. Many of these studies have reported an improvement in students' understanding of their individual professional roles and identities, the roles and identities of other health care professionals, and have improved their perceived value of interprofessional collaboration and education. ${ }^{6-10}$ However, much of the prior work has targeted health care students or professionals in occupational therapy, physical therapy, physician assistants, and nursing. Athletic trainers serve as frontline health care workers providing services for athletic competitions and practices. It is important for athletic trainers to communicate with other health care workers, such as nurses, if athletes in their care require elevation to higher treatment facilities. However, students in athletic training programs remain an understudied population. ${ }^{18}$ Therefore, the purpose of this study was to determine whether students' attitudes had changed when exposed to interprofessional activities and what change was observed.

The study was guided by the following questions:

1. Do students' attitudes of IPE change when exposed to IPE activities?
2. Do students perceive IPE as valuable?

3. Do students' perceptions of the other health care professionals change?

\section{DESIGN, SETTING, AND PARTICIPANTS}

The study was a convivence sample, mixed methods survey design and was conducted during the spring 2020 semester (February-May 2020) at the host institution. Participants included adult college students older than age 18 years who participated in the master's in athletic training (MAT) and doctor of nursing practice (DNP) programs, including family nurse practitioner (FNP) and nurse midwifery (NM) specialty tracks.

The number of potential participants for the research study totaled 28 students (MAT $=11, \mathrm{FNP}=11, \mathrm{NM}=6)$. Students were informed of the interprofessional course requirements as well as the opportunity to participate in the study by completing 2 surveys before and after the IPE activities. Researchers gained university institutional revenue board approval, and all students who participated provided informed consent.

The original study design included 3 IPE activities. To complete the activities, students were assigned to 1 of 11 groups and remained in those groups for all IPE activities. The first IPE activity was an interview, the purpose of which was for students to become acquainted with group members and gain insight into the roles and responsibilities of a practicing individual in another health care field. The second IPE activity was a face-toface immersion event. Day 1 consisted of classroom lectures, skills demonstrations, and case studies. Day 2 for MAT students consisted of performing objective-structured clinical examinations alongside and in collaboration with FNP and NM students in a mock primary care clinical setting. The third IPE activity was a follow-up interview to recap the IPE activities.

Due to COVID-19 restrictions, a deviation from the 2-day, face-to-face event occurred. Faculty from the MAT and DNP programs quickly redesigned the immersion from face-to-face delivery to an online format through CANVAS-a learning platform used by the university. Topics that transferred to the online format included electrocardiogram reading, suturing, interpreting laboratory test results, diagnostic testing, and interpreting radiograms.

Established groups were assigned case studies that had previously been developed for the objective-structured clinical examinations event. Case study topics included diabetes, hypertension with hyperlipidemia, and asthma. The students were given the same case studies and were asked to complete a focused physical examination on a friend, partner, or coworker, then upload a 10-minute video of their physical examination onto a CANVAS discussion board. The physical examination was to be performed in the manner associated with each student's respective specialty and appropriate for each assigned case study based on the patient's chief concern. Each group member was asked to watch their peers' examinations and provide feedback.

Following the video reviews, students conducted a 15- to 30-minute interview with group members, which was recorded and uploaded onto the CANVAS discussion board. The interview included 9 questions for students to address. The first 5 questions focused on students working together to create a care plan for the patient in the assigned case study. Then, students discussed the physical examination process as well as similarities and differences between their specialties. The final 4 questions focused specifically on IPE, which were designed to facilitate discussion around the integration of interprofessional collaboration in clinical practice, including how perceptions of each discipline changed and whether there was benefit or hinderance during the IPE event.

\section{IMPLEMENTATION AND ANALYSIS}

Prior to the IPE activities, an email was sent to each student that included an invitation to participate, information regarding 
the purpose of the study, a link to the pre-activity questionnaire, and how the information from the questionnaire would be used for the study. The email also stated that by clicking the questionnaire link, the student was consenting to participating in the study. If students agreed to participate, they were asked to complete the Readiness for Interprofessional Learning Scale (RIPLS) questionnaire at 2 intervals: once before and once after the IPE events. Students were not required to complete the survey, but they were required to complete the IPE activities since they were graded course assignments.

The RIPLS questionnaire is a validated tool that is used to assess students' attitudes toward IPE. ${ }^{19}$ It is a 2-part questionnaire consisting of a demographic portion and a portion of 19, 5-point scaled items. The demographic portion asked students to provide the first 3 letters of their first and last name, birth year, discipline, sex, and race. Additionally, students were asked to answer whether they had previously completed an RIPLS questionnaire and, if so, how long had it been since completed. The 5-point scaled items are further divided into 3 subscales: teamwork and collaboration, professional identity, and roles and responsibilities.

The first survey was sent prior to any IPE activities. Students were given 3 weeks to complete the survey. Weekly reminders were sent at the beginning of each week. After the final interviews were completed, the second RIPLS survey was sent. Information about the purpose of the study was restated. Students were given 3 weeks to complete the survey, with a weekly reminder sent each Monday.

The quantitative analysis was performed using the RIPLS survey subscales. Variables were tested for normality using the Shapiro-Wilk test. Normally distributed variables were compared between pre- and post-intervention using dependent t-tests, while nonnormally distributed variables were compared using Wilcoxon signed rank tests. Missing data were handled using pairwise deletion. The significance level was set to $p<.05$, a

\section{Table. Demographic Characteristics of Respondents to Both Surveys}

\begin{tabular}{l|l|l|l} 
& TOTAL & MAT & FNP/CNM \\
\hdashline Total number of respondents $(n=$ men $)$ & $23(n=20)$ & $10(n=3)$ & $7(n=0)$ \\
\hdashline $\begin{array}{l}\text { Mean age in years } \pm \text { standard deviation } \\
\text { Number of respondents with prior experience }\end{array}$ & $28.1 \pm 7.0$ & $25.0 \pm 1.9$ & $32.6 \pm 9.2$ \\
with IPE & 7 & 3 & 4
\end{tabular}

priori. All analyses were conducted using SPSS Statistics 26.

The researchers listened to and transcribed the final interviews of the student groups. Once the transcriptions were complete, the researchers read through the transcripts, noting any words or phrases that consistently appeared. After that, they identified common themes that were relevant to interview questions. Several rounds of coding were completed until saturation was reached. Responses were then grouped and categorized into the themes and subthemes.

\section{RESULTS}

The RIPLS survey was used to answer research question one: "Do students' attitudes of IPE change when exposed to IPE activities?"
A total of 23 students had participated in at least 1 survey. Of those 23 , a total of 17 had completed both the pre- and post-intervention surveys. Demographic characteristics of those who had completed both surveys are listed in the Table. Survey responses between MAT and DNP students were not significantly different; therefore, results from the entire group are presented together, as well as separately.

\section{Teamwork and Collaboration}

The mean teamwork and collaboration score at baseline was 41.7 (out of 45.0) for the entire group, indicating that participants had already viewed themselves as skilled in this area. Following the intervention, scores increased slightly to 42.8 . However, this improvement was not statistically significant $(Z=-1.62, p=.05)$ (Figure 1$)$.

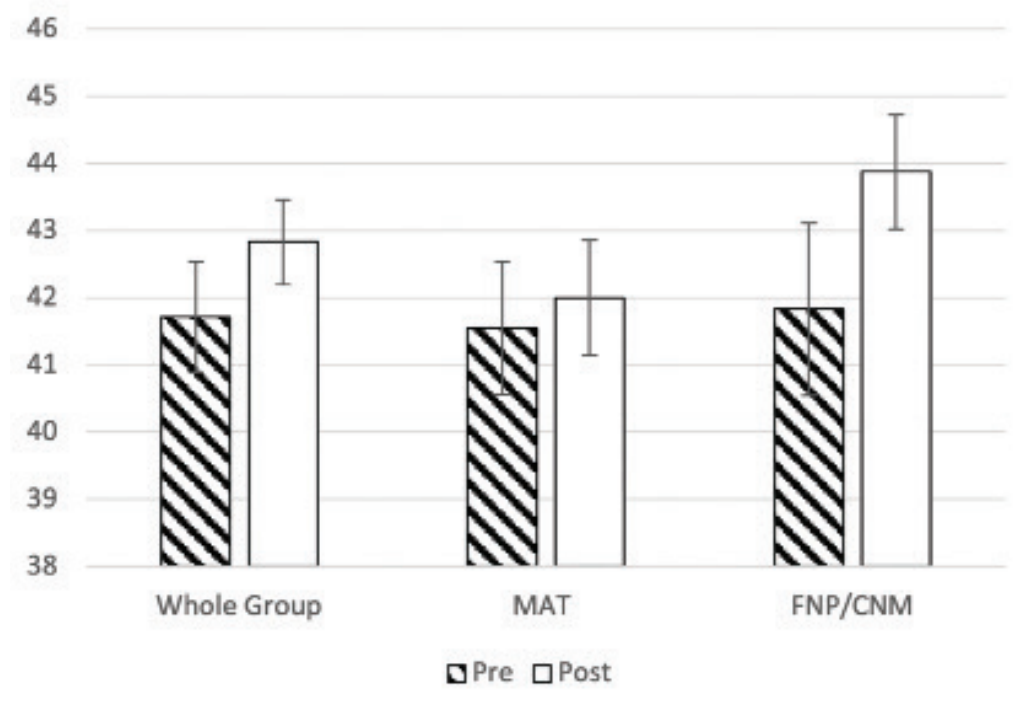

Figure 1. Teamwork and collaboration subscale. Data are presented as mean \pm standard error of the mean 


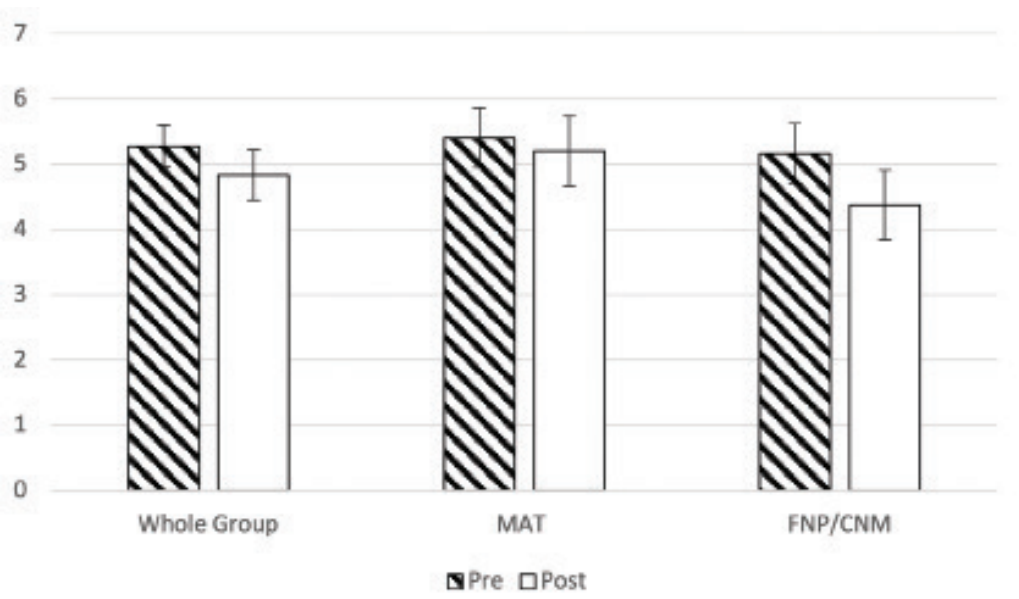

Figure 2a. Negative professional identity subscale. Data are presented as mean \pm standard error of the mean.

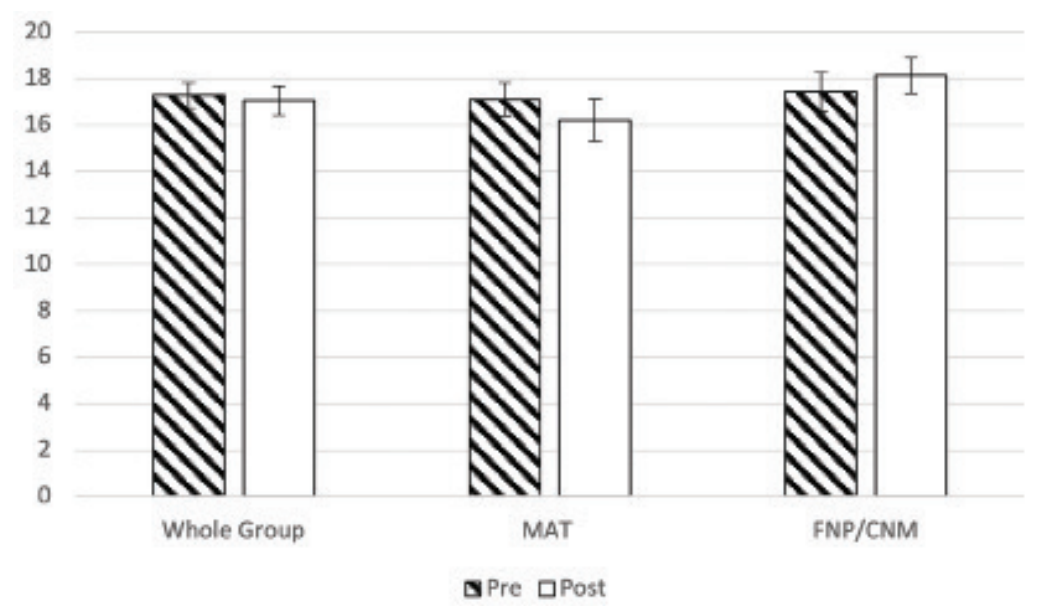

Figure $\mathbf{2 b}$. Positive professional identity subscale. Data are presented as mean \pm standard error of the mean.

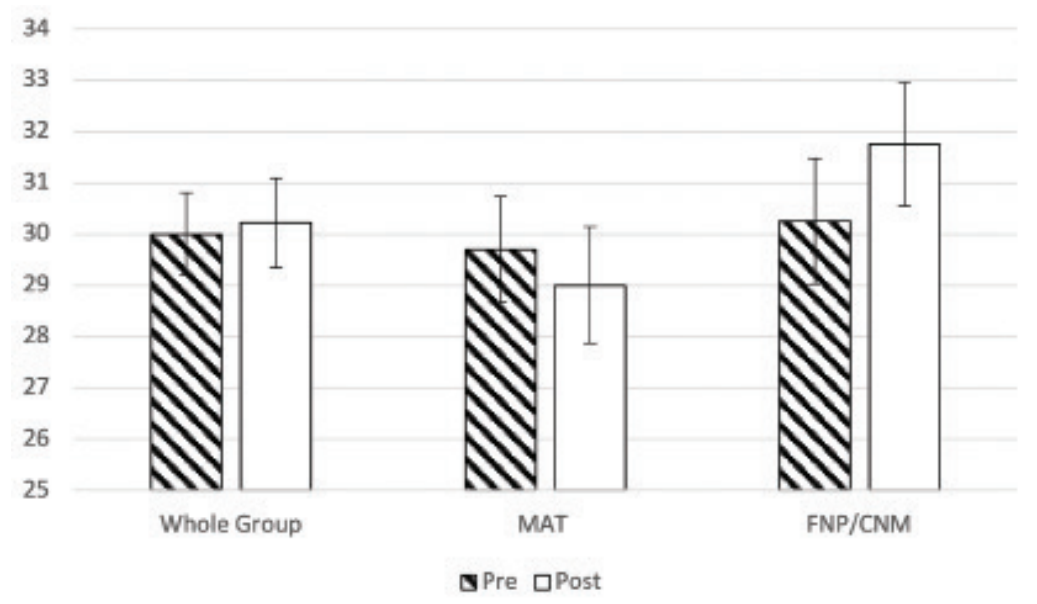

Figure 2c. The full professional identity subscale is a combination of both negative and positive professional identity scales. Data are presented as mean \pm standard error of the mean.

\section{Professional Identity}

The professional identity subscale was analyzed as a whole and separated into negative and positive subcategories. Negative professional identity scores had decreased during the intervention, from 5.2 to 4.8 out of 15 , indicating that participants viewed interprofessional interactions more positively after the intervention. However, this change was not statistically significant $(t=1.41, p=$ .09, Figure 2a).

Positive professional identity scores were relatively unchanged, moving from 17.3 to 17.1 out of 20 . However, this change was not statistically significant $(t=0.54, p$ $=.30$, Figure $2 \mathrm{~b}$ ). When combined, the full professional identity score had increased slightly from 30.0 to 30.2 out of 35 , suggesting that participants were more likely to find value in interprofessional relationships as a result of the intervention. However, this change was not statistically significant $(t=-0.34, p=.40$, Figure $2 c)$.

\section{Roles and Responsibilities}

For the roles and responsibilities subscale, we observed very little change during the intervention, with the subscale score changing from 6.4 to 6.3 out of 15 . indicating that participants had become more confident in their role in the health care process during the intervention. However, this change was not statistically significant ( $t=1.34, p=10$, Figure 3 ). The low baseline score indicates that the participants were already confident in their role prior to beginning the intervention.

To answer research questions 2 and 3-"Do students perceive IPE as valuable?" and "Do students' perceptions of the other health care professionals change?"-students were asked to conduct an interview with each other to discuss if and how their perceptions had changed after completing the IPE activities. There were 11 groups and 28 students; however, coding was only completed for 9 groups and 24 students. One group's interview was excluded because of inaudible quality of the recording, and 1 group did not complete the interview. 


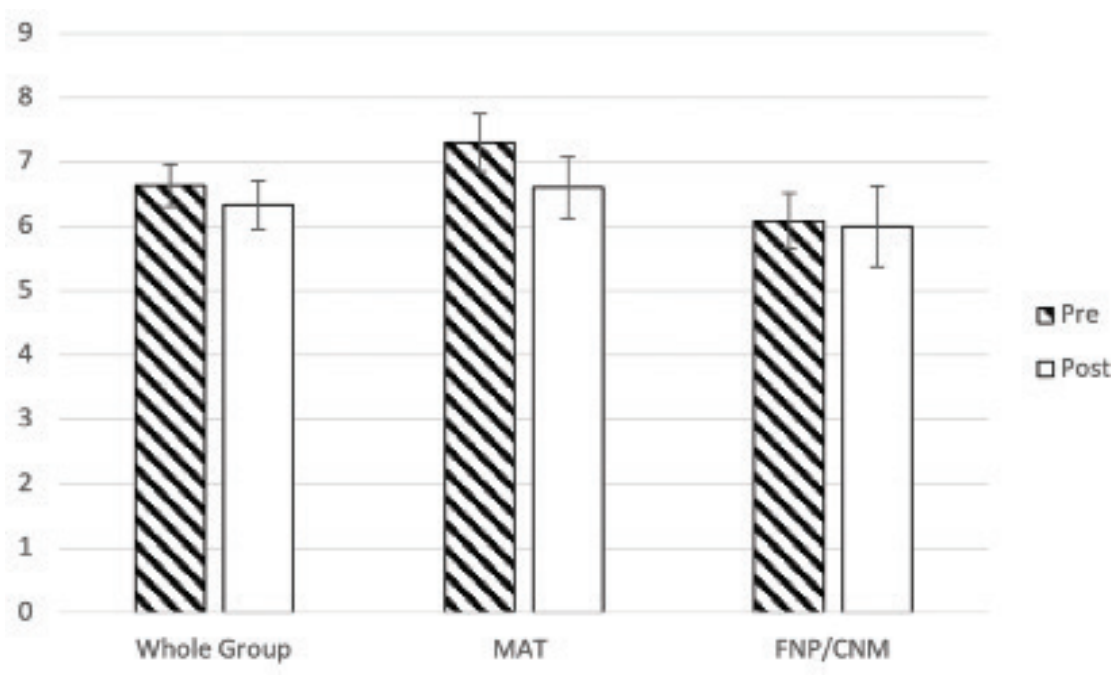

Figure 3. Roles and responsibilities survey subscale. Data are presented as mean \pm standard error of the mean.

Analysis of the 9 group interviews had revealed that participants had perceived the IPE activities as valuable. Participants expressed that exposure to IPE can translate and be beneficial in clinical practice through interprofessional collaboration. The overarching theme that all groups agreed upon was that IPE increases patient-centered care via the following subthemes.

Resources. Seven of the 9 groups had indicated that interprofessional collaboration provided more resources in care for the patient. Students spoke about how working with other professionals provided multiple insights and perspectives. Everyone on the health care team possesses specific knowledge that can be utilized to enhance the quality of patient care. For instance, it was acknowledged that athletic trainers may have more insight relating to changes in baseline information because of the amount of time spent with their patients, whereas nurse practitioners have more knowledge and understanding of the body systems. Additionally, it was expressed that working interprofessionally expanded one's own knowledge and provided opportunities for professional development.
Education of roles and responsibilities. Five of the 9 groups indicated that IPE is valuable because it provides opportunities to learn about the roles and responsibilities of other health care professionals prior to working clinically. Participants expressed an appreciation for having new insight about how other health professionals deliver care and how their expertise could be used in their respective professions when engaging in and providing patient care.

Communication. Four of the 9 groups expressed that communication is increased when practicing interprofessionally. Communication becomes more effective since students learn the professional language used by differing providers and because students then can practice engaging in the language prior to a real-world event. Additionally, communication is enhanced when providers can speak directly to one another rather than depending on patients to convey the information, thus creating a scenario where all providers are working from a place of unity. In the same spirit, students indicated that more effective communication could increase patient satisfaction by reducing confusion, since the patient would not be shuffled between providers.
Analysis of the 9 group interviews revealed that all members' perceptions changed to a degree. The degree of change was predominantly among FNP and NM students and ranged from "no awareness of athletic training as a discipline" to understanding it one way and then arriving at a different conclusion. Additionally, themes had arisen indicating the way in which student perceptions had changed.

Breath of Knowledge. Seven of the groups had indicated that their perceptions had changed in understanding the amount of knowledge that was learned or needed to function as the other health care provider. Prior to the IPE training, many of the FNP and NM students had believed that athletic trainers only functioned orthopedically and expressed not knowing that athletic trainers could perform assessments of illness nor the extent to which they could perform an assessment.

Specifically, NP students had noted that they were surprised that MAT students knew and understood the physical systems and were able to perform assessments of vital signs; the ear, nose, and throat; and the abdominal quadrants. Others had indicated that they were not aware that athletic trainers could help manage patients with conditions such as asthma or diabetes.

MAT students had indicated a realization of the depth of knowledge FNP and NM students needed to conduct a physical examination. MAT students were not aware of the number of systems that were reviewed regardless of the patient's chief concern.

Similarities. All 9 groups had commented on the overlap of skills necessary to function as a health care professional. They indicated that the assessments were similar in terms of the information collected, but the focus and approach was different for their specialties.

Additionally, MAT and DNP students had recognized that a great deal of time is spent educating other individuals, such as community members and patients, 
about the profession in which they practice, since many are unaware of FNP, $\mathrm{NM}$, or athletic training scopes of practice. Students from both the MAT and DNP programs noted that gaining respect as a functional member of a health care team can be difficult at times. Participants had concluded that the lack of understanding of roles and responsibilities can lead to the underutilization of their health care area.

Relationships. MAT and DNP students had noted the differences in rapport and relationships established based on the roles of the health care professional. Many DNP students were surprised at the amount of time that athletic trainers spend with their patients and expressed how this may positively affect the relationships built between each discipline. Students had also noted the advantage in seeing patients on a more-frequent basis and how that experience might enhance their own practice when working collaboratively with athletic trainers.

\section{DISCUSSION}

Overall, the qualitative and quantitative results indicate a positive change in attitudes toward IPE and interprofessional practice.

The RIPLS survey was used to answer research question 1 . While the findings were not statistically significant, there were still positive outcomes. In part, the lack of statistical significance may be due to students already having a positive attitude toward IPE, as indicated by the high scores in the subcategories of the pre-event survey. The results of the postevent survey indicated slight positive changes. For example, the subcategory of teamwork and collaboration indicated the greatest increase in scores, which increased from 41.7 to 42.8 . This positive change supports research that demonstrates exposure to IPE increases the ability to work as a team and increases the value of working as a team member $^{6-10}$ Additionally, there was a change in negative professional identity, indicating that the exposure to the IPE activities had a positive impact on respondents' identity.
Qualitative results support the positive change demonstrated in the quantitative results and provide some specific insight into the degree and type of change that occurred. Results demonstrated that participants perceive IPE as valuable during their education and in supporting their transition into the health care industry. Overall, participants had indicated that IPE would ultimately increase patientcentered care in the following ways:

1. By experiencing and participating in IPE, the resources available to them had increased.

2. Resources are in the form of the knowledge and insight provided by other health care professionals.

3. If individuals with differing health care backgrounds work synergistically to solve a problem, a more comprehensive and holistic approach could be taken for delivering care.

4. Fewer errors would occur, and more patient issues would be identified.

Additionally, participants indicated that communication would improve patient care in the following ways:

1. When working with other health care professionals, one gains an understanding of the different professional languages. This understanding may lead to fewer communication errors.

2. In a truly interprofessional health care team, communication is enhanced because providers speak directly with one another rather than depending on patients to convey pertinent information between various appointments. Improved communication creates a scenario where all providers are working from a place of unity.

3. More effective communication could increase patient satisfaction by reducing confusion, since the patient would not be shuffled between providers.

4. Participants had expressed an appreciation for having opportuni- ties to learn about and work with students in other areas of health care prior to transitioning into practice. They believed providing students with such opportunities was important because it allowed them to learn the roles and responsibilities of other health care providers while in school and prior to transitioning into practice. With this training and understanding, they could better and more effectively use the resources of the health care professionals in their patient care.

Results also indicated that participants' perceptions of other health care professionals had positively changed and that they now view them as individuals who could be part of their health care team with positive impact. The changes were expressed in the following ways:

1. Students had gained an understanding of the type and breadth of knowledge that each health care professional needed to perform their role within the health care team. It was acknowledged that when working together, the partnership could be complementary, since everyone had different knowledge and experiences to lend to each case or situation.

2. Participants' perceptions also changed as related to the types of relationships each professional has with the patient populations for which they serve. Typically, athletic trainers, in a traditional setting such as a university or high school, spend a great deal a time with their patients. FNP and NM students acknowledged how this relationship could benefit their ability to provide patient care, since athletic trainers may have insight into a patient's particular problem that may have otherwise been unknown.

3. Both the MAT and DNP students realized that there were more similarities than differences between 
the 2 professions. Specifically, they experienced an overlap in skills such as patient interactions, conducting physical assessments, and providing patient education. Additionally, they found similarity in the amount of time each spends educating other health care professionals or patients about their professions, as well as the roles and responsibilities each has in providing care to patients.

Again, research has demonstrated positive outcomes when students have opportunities to work with other health care students prior to working in the real world. It seems as though if there is an improvement in understanding the different roles and responsibilities as well as increased effectiveness in communication while students are in school, these skills may ideally translate into the workforce. In turn, these skills may lead to a more-complete transition into practice, reduce the conflict that occurs in practice, and potentially create teams of health care professionals who truly collaborate, leading to more-focused, patient-centered care. This study, while not generalizable, demonstrates that when students have the opportunity to learn with students in other areas of health care, there are positive changes in perceptions of one another and that students find value in IPE, not only while in school but also as they transition into the workforce.

\section{LIMITATIONS}

One limitation of this study is the sample size $(\mathrm{N}=28)$. This was the result of design, as the researchers were specifically targeting students of the 2 courses. It was also optional for students to complete the surveys; for that reason, not all 28 students had completed the surveys, and there was not necessarily equal representation from the different health care areas. Additionally, there were significantly more women than men in the 2 courses, so limited perspective was gained from the male student population.
Also, the researchers used a self-reported survey that may not have resulted in accurate reporting. There are several reasons why results for self-reported surveys may not be accurate. Participants do not always interpret the questions correctly and either skip them or answer in a neutral manner. Additionally, participants may not be interested in the content reasoning behind the research.

The qualitative methodology that was used may have also been a limitation. The researchers had administered open-ended survey questions to be utilized during the recorded group interviews. The inductive process was used until theme saturation was achieved. The qualitative process does induce some bias. It is a process where one reads for words, phrases, or themes that frequently emerge from the data. The same information may produce different results from researcher to researcher.

Finally, situations can change without notice, and health care providers must be able to adapt in an instant, such has been the case for this study. Graduate students enrolled in the MAT and DNP programs had experienced such contrivance in the spring 2020 semester. The IPE in-person activity was canceled because of the stay-at-home orders during the COVID-19 pandemic. With only 1 week of planning, faculty from the MAT and DNP graduate programs quickly turned what was supposed to have been a live event into a virtual experience for their students. Therefore, students were not able to participate in the live IPE event in the way it was intended.

\section{CONCLUSIONS}

This study reports improvement in the RIPLS survey subscales because of an IPE intervention in a sample of MAT and DNP students. Improvement was relatively modest, likely because of the relatively good, preintervention scores. Our qualitative analysis indicated that study participants benefitted from IPE by being able to better understand the scope of practice between different health care professionals and felt that they would be better able to communicate with other professionals in the workplace because of IPE. Athletic trainers and nurses provide the most direct care for individuals. Therefore, future investigations should be aimed at developing IPE programs that benefit both groups and help improve patient care.

\section{References}

1. Barnsteiner JH, Disch JM, Hall L, Mayer D, Moore SM. Promoting interprofessional education. Nurs Outlook. 2007;55(3):144-150. https://doi.org/10.1016/j.outlook.2007.03.003

2. Gilbert JH, Yan J, Hoffman SJ. A WHO report: framework for action on interprofessional education and collaborative practice. J Allied Health. 2010;39(Suppl 1):196-197. https://www.who.int/hrh/resources/framework_action/en/

3. Pursuing and maintaining accreditation of professional programs in athletic trainings: implementation guide to the CAATE 2020 professional standards. Commission on Accreditation of Athletic Training Education. Published July 1, 2020. Accessed May 18, 2021. https:// caate.net/wp-content/uploads/2020/11/ Pursuing-and-Maintaining-Accreditation-AND-Guide-to-2020-Standards-FInal_Sept-2020.pdf

4. The essentials of doctoral education for advanced nursing practice. American Association of Colleges of Nursing. Published October 2006. Accessed May 18, 2021. https://www.aacnnursing.org/Portals/42/ Publications/DNPEssentials.pdf

5. Core competencies for interprofessional collaborative practice: 2016 Update. Interprofessional Education Collaborative. Published 2016. Accessed May 18, 2021. https://ipec. memberclicks.net/assets/2016-Update.pdf

6. Hind M, Norman I, Cooper S, et al. Interprofessional perceptions of health care students. J Interprof Care. 2003;17(1):21-34. https://doi. org/10.1080/1356182021000044120

7. Kim YJ, Radloff JC, Stokes CK, Lysaght CR. Interprofessional education for health science students' attitudes and readiness to work interprofessionally: a prospective cohort study. Braz J Phys Ther. 
2019;23(4):337-345. https://doi.org/10.1016/j. bjpt.2018.09.003

8. Lewis GR, Pole D, Linsenmeyer W, Rahman $\mathrm{R}$, Briggs $\mathrm{E}$, Eliot $\mathrm{K}$. The relationship between interprofessional education experience and perceptions, values, and attitudes towards interprofessional collaboration in dietetics practice. $J$ Interprof Educ Pract. 2020;20:1-5. https://doi.org/10.1016/j. xjep.2020.100346

9. Madigosky WS, Franson KL, Glover JJ, Earnest M. Interprofessional education and development (IPED): a longitudinal team-based learning course introducing teamwork/collaboration, values/ethics, and safety/quality to health professional students. J Inteprof Educ Pract. 2019;16:100220. https://doi.org/10.1016/j.xjep.2018.12.001

10. Michalec B, Giordano C, Pugh B, Arenson C, Speakman E. Health professions students' perceptions of their IPE program: potential barriers to student engagement with IPE goals. J Allied Health. 2017;46(1):10-20.

11. Olson R, Bialocerkowski A. Interprofessional education in allied health: a systematic review. Med Educ. 2014;48(3):236-246.

https://doi.org/10.1111/medu.12290

12. Clark K, Congdon HB, Macmillan K, Gonzales JP, Guerra A. Changes in perceptions and attitudes of healthcare profession students pre and post academic course experience of team-based 'care for the critically ill' J Prof Nurs. 2015;31(4):330-339. https://doi. org/10.1016/j.profnurs.2015.01.006

13. Zorek J, Raehl C. Interprofessional education accreditation standards in the USA: a comparative analysis. $J$ Interprof Care. 2013;27(2):123-130. https://doi.org/10.3109/1 3561820.2012.718295

14. Athletic training education competencies, 5th ed. National Athletic Trainers' Association. Published 2011. Accessed May 18, 2021. https://www.nata.org/sites/default/files/ competencies_5th_edition.pdf

15. Breitbach AP, Sargeant DM, Gettemeier PR, et al. From buy-in to integration: melding an interprofessional initiative into academic programs in the health professions. $J$ Allied Health. 2013;42(3):e67-e73.

16. Faculty tool kit: the essentials of baccalau- reate education for professional nursing practice. American Association of Colleges of Nursing. Published February 19, 2009. Accessed May 18, 2021. https://www.aacnnursing.org/Portals/42/AcademicNursing/ Tool\%20Kits/BaccEssToolkit.pdf

17. Tool kit: the essentials of master's education in nursing. American Association of Colleges of Nursing. Published December 18, 2017. Accessed May 18, 2021. https://www. aacnnursing.org/Portals/42/AcademicNursing/Tool\%20Kits/Masters-Tool-Kit.pdf

18. Rizzo CS, Breitbach AP, Richardson R. Athletic trainers have a place in interprofessional education and practice. $J$ Interprof Care. 2015;29(3):256-257. https://doi.org/10. 3109/13561820.2014.942778

19. Reid R, Bruce D, Allstaff K, McLernon D. Validating the Readiness for Interprofessional Learning Scale (RIPLS) in the postgraduate context: are health care professionals ready for IPL? Med Educ. 2006;40(5):415-422. https://doi.org/10.1111/ j.1365-2929.2006.02442.x 